\title{
Trials of a New Stirrer-less Juice Sulphiter at Experimental Sugar Factory
}

\author{
Narendra Mohan \& S. K. Trivedi \\ National Sugar Institute \\ Kanpur, India
}

\begin{abstract}
In India most of the sugar factories produce plantation white sugar by Double Sulphitation process where the most essential and critical equipment used during the clarification process is "juice sulphiter". The clarification efficiency is very much dependent upon the efficiency of juice sulphiter also which in turn depends upon the design features of juice sulphiter. Authors have tried one such juice sulphiter which is also considered as energy efficient at the Experimental Sugar Factory of the Institute with satisfactory results.
\end{abstract}

Keywords:- Plantation white sugar, clarification, energy.

\section{INTRODUCTION}

During the sugar season 2019-20, 457 sugar factories are in operation in the country and the expected sugar production is likely to be about 26.8 million tons. During the previous year 2018-19, 527 sugar factories operated \& produced about 33.2 million tons of sugar. About $95 \%$ of sugar so produced is plantation white sugar manufactured by double sulphitation process of clarification.

As is well known, in double sulphitation process, raw juice is heated up to about $70^{\circ} \mathrm{C}$ and then is sent to juice sulphiter for liming and sulphitation, $\mathrm{pH}$ of sulphited juice is maintained around 7.0-7.1. The sulphited juice is again heated up to $102-103^{\circ} \mathrm{C}$ and sent to clarifier for settling, after settling, clear juice is sent for further processing. Juice sulphiter is the vital clarification equipment for determining precipitation of the non-sugars present in the juice by ensuring proper admixing of reactants i.e. milk of lime and sulphur dioxide gas with juice, side by side ensuring that precipitate formation is not disturbed. Being a vital equipment of the processing house, continuous efforts have been made to improve the design features so as to have better juice sulphitation as reflected from minimum change in reducing sugar $/ 100^{\circ}$ brix, higher colour removal and purity rise etc. Efforts have also been made to develop and use stirrerless juice sulphiters to save on energy without disturbing floc formation. Authors designed and tried one such sulphiter at Experimental Sugar Factory of National Sugar Institute which accomplishes the task with lower energy requirements.

\section{VARIOUS DESIGNS OF JUICE SULPHITERS}

A continuous development in the design features of juice sulphiters has taken place on the basis of observations made on their working. They may be stirrer or stirrer less or may differ in system of adding milk of lime i.e. pre liming, shock liming or simultaneous addition of milk of lime and sulphur dioxide gas. Various such designs of juice sulphiters are in use, some of them, which are being widely used, are discussed here below.

\section{A. Nsi Design Juice Sulphiter}

One of the most commonly used and adopted design in the sugar industry for simultaneous liming and sulphitation is known as NSI design. It is a cylindrical tank in which another inverted cylindrical shell closed from top is suspended. Suitable stirrers are driven through a central shaft which in turn is driven by a motor and reduction gear. Juice after passing through the absorption tower is sprayed over the perforated plane where squeezers are provided for keeping perforation clean. Milk of lime enters in to the tank where gas enters through perforated coil. Depending upon the diameter of the tank more than one milk of lime entries is provided at equal spacing for uniform lime distribution. The distance between the gas coil and milk of lime entry is essential and is generally kept 200- $250 \mathrm{~mm}$. After treatment, the juice overflows out of the tank through over flow pipe. In this design of the juice sulphiter, moving and static mixers are provided in such a way to facilitate mixing of lime, juice and gas without affecting the precipitate formed. Now a day's gas coils generally made of SS 304 are provided so as to have trouble free and efficient working of the sulphiter.

\section{B. Sparger Type Juice Sulphiter With Stirrer}

A Sparger type juice sulphiter but having a small stirrer for juice and milk of lime mixing has reported to be worked well in the sugar industry. In this design also milk of lime connection is so made that entire lime comes in contact immediately with juice entering the main vessel and after 56 seconds comes in contact with $\mathrm{SO}_{2}$ gas. The juice sulphitation system otherwise as a whole comprises of pre and shock liming arrangement for use, as and when, required. For ensuring complete mixing of lime with juice, a stirrer has been fitted at the bottom of the sulphiter by providing double gland system, so that, any leakages of juice from drive end do not occur. 


\section{Ejector Sulphitation System}

In an ejector sulphitation system, which is a modified version of "Quarez System" in the literature has also been used by few sugar factories for juice sulphitation. The system is claimed to be of a more compact design, needs less steam, uses less power and hence is considered more energy efficient. Such system is working satisfactorily in one sugar factory in India where in sulphur melts by its own heat of combustion and there is no need of medium pressure steam. The sulphur burner uses induced draft air thus eliminating the air blower and compressor.

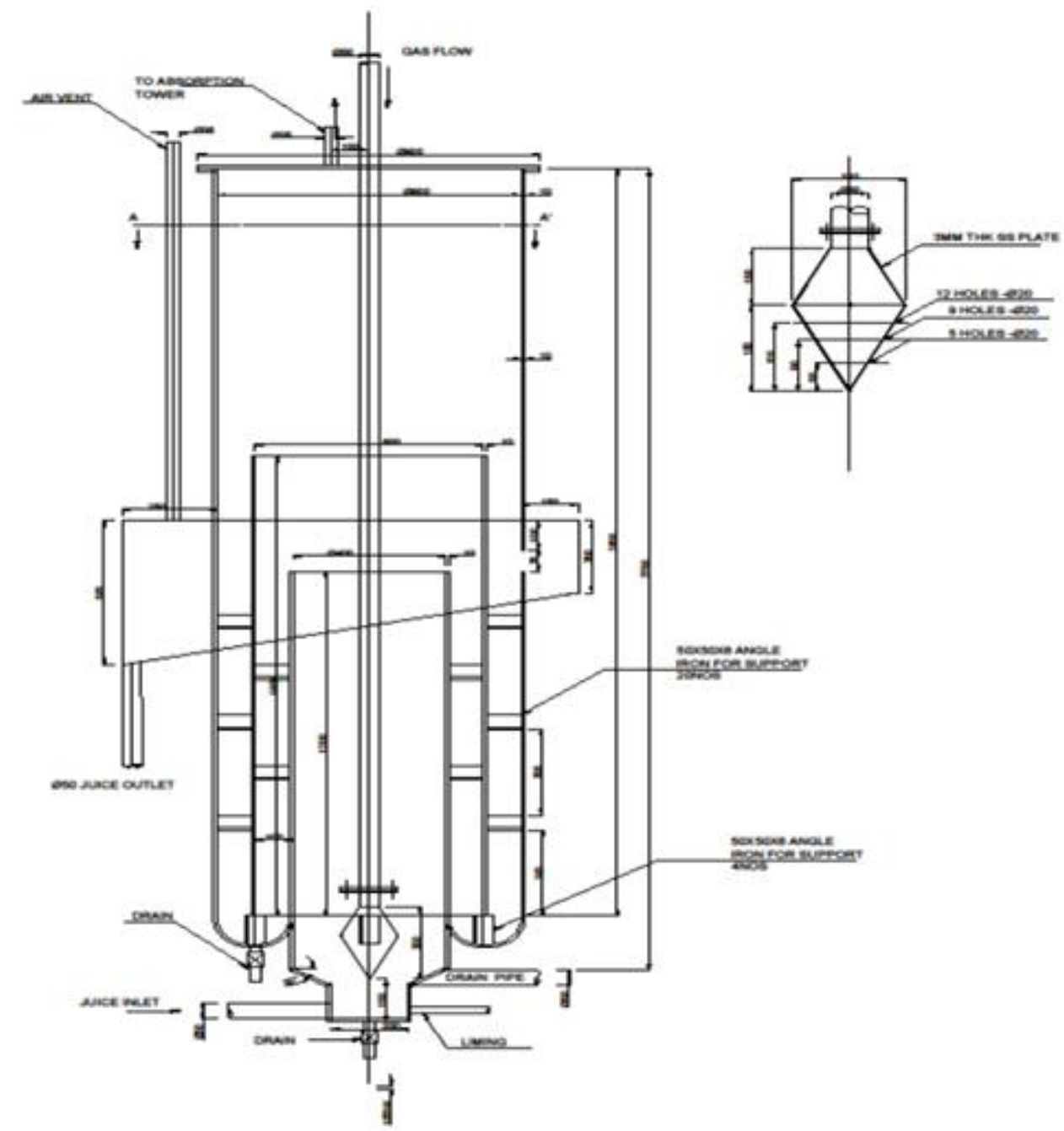

Fig 1:- NSI Stirrer-less Juice Sulphiter

\section{MATERIAL AND METHODS}

The mixed juice and clear juice samples were analyzed for brix and purity on Sucro Analyzer as per standard protocol at 589 $\mathrm{nm}$, reducing sugar per $100^{\circ}$ brix by Lane \& Eynon method, $\mathrm{CaO}$ content by EDTA method and the colour of juices were determined on Sucroscan Colour Analysis Kit by ICUMSA method GS-2/3-10. The samples for other parameters were analyzed as per ICUMSA and Standard Indian protocols. The trials were conducted during the crushing season for 3 days from $1^{\text {st }}$ March, 2020 to $3^{\text {rd }}$ March, 2020. Its working was compared with the existing NSI design juice sulphiter available in the Experimental Sugar Factory. The details of analysis of mixed and clear juice for various parameters are given in the following tables: 
ISSN No:-2456-2165

\begin{tabular}{|c|c|c|c|c|c|c|}
\hline & \multicolumn{3}{|c|}{ MIXED JUICE } & \multicolumn{3}{c|}{ CLEAR JUICE } \\
\hline Date & Bx. \% & Purity & $\begin{array}{c}\text { CaO content } \\
(\mathbf{p p m})\end{array}$ & Bx. \% & Purity & $\begin{array}{c}\text { CaO content } \\
(\mathbf{p p m})\end{array}$ \\
\hline $1-3-2020$ & 12.87 & 79.62 & 730 & 12.90 & 80.02 & 1110 \\
\hline & 13.10 & 80.20 & 790 & 13.18 & 80.72 & 1220 \\
\hline & 12.60 & 78.90 & 750 & 12.72 & 79.50 & 1200 \\
\hline & 12.90 & 79.76 & 710 & 12.98 & 80.42 & 1160 \\
\hline $2-3-2020$ & 13.24 & 80.30 & 680 & 13.30 & 80.99 & 1130 \\
\hline & 13.15 & 80.10 & 710 & 13.19 & 80.81 & 1140 \\
\hline & 13.45 & 80.49 & 830 & 13.48 & 81.19 & 1250 \\
\hline $3-3-2020$ & 13.20 & 81.20 & 820 & 13.23 & 81.02 & 1270 \\
\hline & 13.10 & 81.40 & 820 & 13.16 & 81.95 & 1230 \\
\hline & 12.64 & 80.62 & 800 & 12.70 & 81.10 & 1240 \\
\hline & 12.96 & 81.59 & 670 & 12.98 & 81.99 & 1180 \\
\hline & 13.06 & 82.42 & 690 & 13.10 & 83.07 & 1100 \\
\hline
\end{tabular}

Table 1:- Analysis of mixed juice and clear juice for $\mathrm{Bx} . \%$, Purity and $\mathrm{CaO}$ content in ppm

\begin{tabular}{|c|c|c|c|c|c|c|}
\hline & \multicolumn{3}{|c|}{ MIXED JUICE } & \multicolumn{3}{c|}{ CLEAR JUICE } \\
\hline Date & Bx. \% & Purity & RS/ 100 $\mathbf{B x}$ & $\mathbf{B x}$ \% & Purity & RS/ 100 $\mathbf{B x}$ \\
\hline $1-3-2020$ & 12.87 & 79.62 & 5.38 & 12.90 & 80.02 & 5.34 \\
\hline & 13.10 & 80.20 & 5.34 & 13.18 & 80.72 & 5.19 \\
\hline & 12.60 & 78.90 & 5.57 & 12.72 & 79.50 & 5.64 \\
\hline & 12.90 & 79.76 & 5.38 & 12.98 & 80.42 & 5.06 \\
\hline $2-3-2020$ & 13.24 & 80.30 & 5.75 & 13.30 & 80.99 & 6.01 \\
\hline & 13.15 & 80.10 & 5.59 & 13.19 & 80.81 & 5.42 \\
\hline & 13.45 & 80.49 & 5.76 & 13.48 & 81.19 & 5.62 \\
\hline & 13.20 & 81.20 & 6.05 & 13.23 & 81.02 & 5.90 \\
\hline & 13.10 & 81.40 & 6.27 & 13.16 & 81.95 & 6.20 \\
\hline & 12.64 & 80.62 & 6.41 & 12.70 & 81.10 & 6.16 \\
\hline & 12.96 & 81.59 & 6.19 & 12.98 & 81.99 & 6.33 \\
\hline & 13.06 & 82.42 & 6.12 & 13.10 & 83.07 & 5.70 \\
\hline
\end{tabular}

Table 2:- Analysis of Reducing sugar /1000Brix in mixed juice and clear juice

\begin{tabular}{|c|c|c|c|c|c|c|c|}
\hline \multirow[b]{2}{*}{ Date } & \multicolumn{3}{|c|}{ MIXED JUICE } & \multicolumn{3}{|c|}{ CLEAR JUICE } & \multirow[b]{2}{*}{$\begin{array}{c}\text { \% Reduction in } \\
\text { Colour }\end{array}$} \\
\hline & Вx. \% & Purity & Colour (IU) & Bx. \% & Purity & Colour (IU) & \\
\hline \multirow[t]{4}{*}{$1-3-2020$} & 12.87 & 79.62 & 31532 & 12.90 & 80.02 & 24220 & 23.19 \\
\hline & 13.10 & 80.20 & 30956 & 13.18 & 80.72 & 22595 & 27.00 \\
\hline & 12.60 & 78.90 & 31163 & 12.72 & 79.50 & 23036 & 26.08 \\
\hline & 12.90 & 79.76 & 29981 & 12.98 & 80.42 & 23745 & 20.80 \\
\hline \multirow[t]{4}{*}{$2-3-2020$} & 13.24 & 80.30 & 29562 & 13.30 & 80.99 & 20366 & 31.10 \\
\hline & 13.15 & 80.10 & 28673 & 13.19 & 80.81 & 22594 & 21.20 \\
\hline & 13.45 & 80.49 & 28718 & 13.48 & 81.19 & 22555 & 21.49 \\
\hline & 13.20 & 81.20 & 30142 & 13.23 & 81.02 & 24544 & 18.57 \\
\hline \multirow[t]{4}{*}{$3-3-2020$} & 13.10 & 81.40 & 30294 & 13.16 & 81.95 & 24211 & 20.08 \\
\hline & 12.64 & 80.62 & 28912 & 12.70 & 81.10 & 22800 & 21.14 \\
\hline & 12.96 & 81.59 & 29748 & 12.98 & 81.99 & 23114 & 22.30 \\
\hline & 13.06 & 82.42 & 30167 & 13.10 & 83.07 & 20639 & 31.58 \\
\hline
\end{tabular}

Table 3:- Analysis of colour reduction\% from mixed juice to clear juice 


\section{OBSERVATIONS}

In order to assess the efficiency of stirrer less juice sulphiter it was decided to conduct continuous trials for duration of three consecutive days, such that, every day various performance data was collected and analyzed as per table No.1-3. The investigation was limited to the following:

$>$ Apparent purity rise from mixed juice to clear juice

$>$ Destruction of reducing sugars

$>$ Rise in Cao content from mixed juice to clear juice

$>$ Colour reduction from mixed juice to clear juice

\section{A. Purity rise}

It is generally accepted that the extent of purity rise from mixed juice to clear juice is an indicator of good clarification and removal of non- sugars. During the trials the purity rise from mixed juice to clear juice was ranging between 0.40 to 0.70 units. Only in one case decrease in purity from mixed juice to clear juice was observed which was nominal and may be due to intermittent working of factory during the period.

\section{B. Change in reducing sugar content from mixed juice to clear juice}

Higher reducing sugar and ash ratio has a favorable effect on reduction in sucrose purity of final molasses. It is therefore necessary that the destruction of reducing sugars should always be avoided as far as possible. Their destruction also causes colour formation in process and is detrimental to sugar quality. Similarly, rise in reducing sugar content indicates inversion of sucrose, which is also not desirable. The change in reducing sugars per $100^{\circ} \mathrm{Bx}$ during the trials was about $+/-5 \%$, which considered normal.

\section{Rise in CaO Content:}

The increase in $\mathrm{CaO}$ content during liming and sulphitation results in scaling of the evaporators and increase in molasses formation. Therefore, the increase in $\mathrm{CaO}$ content from mixed juice to clear juice was also analyzed during the duration of trials and it was observed that the increase in $\mathrm{CaO}$ content varied from $400-450 \mathrm{mg}$ per liter or increase of about $40 \%$ which is considered to be within limits.

\section{Colour reduction from mixed juice to clear juice:}

The mixed juice colour varied from about 28500 to 31500 IU, while colour of clear juice varied from about 20600 to 24500 IU. The colour reduction from mixed juice to clear juice was observed to be in the range of about 20$30 \%$ which on an average may be considered about $25 \%$. Such colour reduction is considered satisfactory in double sulphitation process.

\section{CONCLUSION}

The working of newly developed Stirrerless juice sulphiter was observed to be satisfactory as reflected from the analytical results. The working was observed to be better than the conventional juice sulphiter as reflected from the values of purity rise and colour removal. Another important consideration is that this stirrerless juice sulphiter worked well with short retention time clarifier having a retention time of about 45 minutes only. The desired clarity of clear juice and compactness of muddy juice could be obtained.

This stirrerless juice sulphiter besides ensuring better clarification and saving energy while working with short retention clarifier may result in to lower unknown losses and decrease in juice temperature thus lowering steam requirements.

\section{ACKNOWLEDGEMENT}

Authors are thankful to the Ministry Consumer Affairs, Food \& Public Distribution, Department of Food \& Public Distribution for providing us the financial support and cooperation to carry out the study.

\section{REFERENCES}

[1].Book on Clarification of sugar cane juice by Narendra Mohan -2019, page No.73-77. 